\title{
CONF-961102-- 3
}

LA-UR- $96-4039$

\section{Beyonci the Brillouin Limit with the Penning Fusion Experiment}

\author{
D. C. Barnes \\ Applied Theoretical \& Computational Division, \\ Los Alamos National Laboratory \\ T. B. Mitchell, M. M. Schauer \\ Physics Division, Los Alamos National Laboratory \\ RECFIVE \\ FER 141997 \\ OSTI
}

(November 9, 1996)

\begin{abstract}

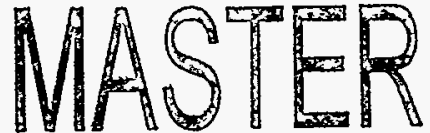

Several years ago, it was proposed that a dense nonneutral plasma could be produced in a Penning trap. Nonneutral plasmas have excellent confinement. Thus, such a dense plasma might produce simultaneously high density and good confinement (as needed for fusion). Recently, this theoretical conjecture has been demonstrated in a small ( $3 \mathrm{~mm}$ radius) electron experiment (PFX). Densities up to 35 times the Brillouin density (limiting number density in a static trap) have been inferred from the observed strong (100:1) spherical focussing. Electrons are injected at low energy from a single pole of the sphere. A surprising observation is the self-organization of the system into a spherical state, which occurs precisely when the trap parameters are adjusted tc produce a spherical well. This organization is observed by a bootstrapping which produces a hysteresis. Additional observations which confirm the dense spherical focus are energy-scattered electrons and deflections of an electron probe beam by the space charge of the central focus.
\end{abstract}

PACS: 52.75.-d, 52.58.Qv

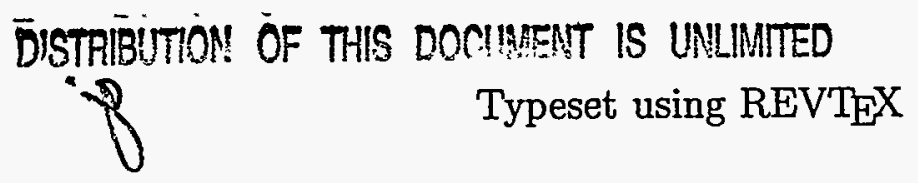




\section{DISCLAMMER}

Portions of this document may be illegible in electronic image products. Images are produced from the best available original document. 


\section{INTRODUCTION}

This paper reports on recent experimental $[1,2]$ and theoretical work which is exploring the applicability of nonneutral plasma confinement for the production of dense, wellconfined, thermonuclear plasmas. In contrast to quasineutral, magnetically-confined laboratory plasma devices, Penning traps [3] have exhibited remarkable confinement of nonneutral plasmas. It is natural to ask whether such excellent confinement might be useful for controlled thermonuclear fusion. A first, obvious, and fundamental difficulty is the density limit associated with unneutralized space charge.

Due to the charge nonneutrality of these plasmas, the densities attainable in static traps are limited, the maximum value being the Brillouin limit [4].

$$
\bar{n}=\frac{B^{2}}{2 \mu_{o} m c^{2}}
$$

where $B$ is the magnetic induction, $\mu_{o}$ the permeability of free space, $m$ the mass of confined particles, and $c$ the speed of light.

It was suggested [5] recently that the local density might exceed $\bar{n}$ in a strongly nonequilibrium plasma. This local concentration may occur either in space or time. Here, the possibility of spatial concentration via spherical focussing is studied. In this approach Penning trap confinement is combined with spherical focussing to produce a dense, quasineutral, thermonuclear core plasma. The concept of spherical focussing of ions was studied by Hirsh [6] in a system in which a grid provided ion confinement and acceleration. More recently, Bussac and Krall [7] introduced the concept of Spherically Convergent Ion Focus (SCIF) systems. Their work first introduced the important concept of using electrons confined by a combination of magnetic and electric fields to produce a large negative space charge field for the gridless acceleration and confinement of spherically convergent ions.

Nonneutral plasma confinement is made useful for fusion by combining several new features of operation with previous nonneutral experience. To obtain a reasonable average density with achievable magnetic induction limits, electrons are chosen as the nonneutral 
confined species. In this case, the technological limit becomes electrostatic breakdown associated with the space charge field of a moderate density electron plasma. Next, the moderate electron density is concentrated in space in one of two ways. First, a nonthermal electron velocity distribution may be produced by arranging a throughput of electrons which maintain a spherically-convergent beam distribution. Second, a uniform electron density may be used to confine ions with a nonthermal, SCIF beam distribution. While collision rates indicate that the latter approach is preferred for the final system, the former is simpler, both conceptually and experimentally, and is the one investigated here. Finally, electron space charge provides confinement of a dense, thermonuclear core ion plasma in which fusion reactions may occur.

Several physics and technology issues are associated with spherical focussing schemes. Limitations of electrical breakdown have already been mentioned. Coulomb collisions $[8,9]$ may require the power to sustain a nonthermal electron or ion distribution to be so large that high $Q$ (thermonuclear gain) is unachievable. Electrostatic or other instabilities may destroy the configuration of make it impractical to sustain. Such a spectrum of difficulities is typical of fusion systems, of course. This paper does not address all or most of these difficulties, but rather presents only the first experimental step in addressing these issues, supporting theory and modeling, and indicates the most immediate next steps which should be considered.

To examine the feasibility of spherical focussing in the simplest nonneutral plasma, a small, low-power, electron machine has been built and operated. A nonthermal, convergent electron distribution is observed to produce strong focussing when parameters are so arranged as to produce a spherical system. A large density enhancement is associated with this focussing. The observations and theory supporting their interpretation are presented here.

The remainder of this paper is organized as follows. In the next Section, the experimental arrangement is described. Section 3 presents the experimental observations, which are analyzed in the subsequent Section. A final Section discusses this work and presents 
conclusions and a brief jummary of near-term future plans.

\section{EXPERIMENTAL ARRANGEMENT}

In its simplest form, a Penning trap consists of three electrodes, the inner surfaces of which are hyperbolae-of-revolution [10]. Two end cathode caps and a central anode ring, (see Fig. 1) produce a rotationally symmetric electrostatic quadrupole field when a voltage, $V$, is applied. Charged particles are confined axially in the electrostatic well, while radial confinement is provided by a uniform magnetic field applied parallel to the trap axis. As previously proposed, [5] the eccentricity of the resulting spheroidal well is tunable, and the well becomes spherical at a particular combination of $B$ and $V$. Details of this aspect variation are discussed in the subsequent Section.

Several design features have been incorporated into PFX to allow the highest applied voltage, and associated highest electron density. First, the trap electrodes are fashioned from titanium due to its superior vacuum and high voltage characteristics. Second, highpurity, chromium-oxide coated alumina insulators provide the mechanical alignment of the three electrodes. The choice of insulator material and careful tailoring of the electric stress near the cathode ends of these insulators by shaped alignment pins provides extremely high resistance to insulator flashover. Finally, the entire trap is operated at liquid $\mathrm{He}$ temperature to provide a vacuum of better than $10^{-10} \mathrm{~T}$ orr and to passivate the electrode surfaces.

These features allow routine operation up to $10 \mathrm{kV}$ applied anode voltage. Actually, voltages up to $30 \mathrm{kV}$ may be supported by the present PFX front-end, when the entire assembly is at cryogenic temperature. This standoff is degraded, apparently by the heat load associated with the present close proximity of the electron emitter (discussed subsequently) to the lower cathode.

The inner diameter of the anode ring is $6 \mathrm{~mm}$ as is the minimum separation of the two end cathode caps. Both of the end cathodes are pierced through the center by $400 \mu \mathrm{m}$ diameter holes which allow injection of electrons into the trap via the lower end cathode 
while providing diagnostic access through the upper one. Low angular momentum electrons are provided by a $L a B_{6}$ crystal emitter situated a short distance behind the hole in the lower end cathode (Fig. 1). The emitter consists of a cone with 60 degree included angle ending in a $200 \mu m$ diameter flat surface. Most of the emission originates on this flat surface, thereby producing a roughly uniform, $200 \mu m$ diameter beam.

The trap assembly is contained within a stainless steel vacuum vessel that is inserted into the bore of a superconducting magnet capable of producing fields up to $6 T$. As the magnet is operated in cold bore configuration, the trap is held at liquid helium temperature. Typical operating voltages range from $500 \mathrm{~V}$ to $10 \mathrm{kV}$ with corresponding magnetic fields necessary to produce a spherical well ranging from $0.05 \mathrm{~T}$ to $0.22 \mathrm{~T}$.

Above the trap, in the fringe of the magnetic field, is located a microchannel plate/phosphor screen (MCP) assembly. Electrons can be transported from the trap through the hole in the upper end cathode and two independently biasable drift tubes to the MCP. The MCP can be used in particle counting mode by means of a multichannel scaler or imaging mode by capturing phosphor screen images with a CCD camera and frame grabber.

Typical operation is as follows. First, the magnetic field is set to the desired value, and the voltages applied to the upper and lower end cathodes are set to their nominal values, usually within a few tens of volts of ground. Next, the anode voltage, $V$, is ramped to the desired value and electron current into the trap, $I_{i}$, is started. Usually, the electron beam is reflected back upon itself by biasing the upper end cathode more negatively with respect to ground than the emitter crystal, although some exceptions will be mentioned later.

Measurements of currents to various electrodes can then be made. Total current scattered from the beam to the anode, $I_{A}$, may be measured, and electrons scattered up in energy, and thus able to climb over the barrier presented by the upper end cathode, may be counted at the MCP. After some predetermined time, $I_{i}$ is turned off and $V$ is ramped to zero. As $V$ is decreased, any electrons which have lost energy and thus remained trapped escape the well and are counted at the MCP. One is now ready to repeat the measurements or move to a new magnetic field value. 


\section{EXPERIMENTAL OBSERVATIONS}

Consider first PFX operation with very low current injected from the electron source. The electron optics are such that a nearly uniform beam will be extracted from the emitter, accelerated by the anode voltage, decelerated again as it approaches the upper cathode, and reflected back without intersecting the upper cathode. Such beam electrons will then reflex near the system axis of symmetry until the electrons drift across the magnetic field and are collected on the lower cathode, near the injection hole.

Next, consider what can occur if higher electron current is injected. In this case, space charge may cause significant deflections of the beam electrons during a single transit. If this deflection is comparable to the beam radius, a fraction of the injected electrons will remain in the trap for many transits, bouncing within the vacuum well and returning near the trap center. The optics of these reflexing beam electrons depends on the selection of $V$ and $B$, which determine the shape of the spheroidal well.

It is easy to show that the effective potential for zero-canonical angular momentum electrons in a combined electrostatic quadrupole and uniform $B$ is

$$
\Phi_{e f f}=\frac{V}{3 a^{2}}\left(r^{2}-2 z^{2}\right)-\frac{e B^{2}}{8 m} r^{2}
$$

where $e$ is the elementary charge, $a$ the trap radius, and $r, z$ are usual cylindrical coordinates. It may be seen from (2) that the particular choice

$$
V_{o}=\frac{e B^{2} a^{2}}{8 m_{e}}
$$

produces a spherical well in which $\Phi_{e f f}$ depends only on the spherical radius $\rho,\left(\rho^{2}=r^{2}+z^{2}\right)$. Further, it may be seen that for $V$ less than $V_{o}$ the well will be prolate, while for values greater than $V_{o}$ the well will be oblate. In the former case, the limiting equipotential will intersect only the cathodes near the system axis, while in the latter case, the limiting surface will intersect the cathodes and a portion of the anode ring.

A remarkable feature of the observations is the spontaneous appearance of a nearly spherically symmetric state precisely when (3) is satisified. Then, if the injection current, 
$I_{i}$, exceeds a threshold current, a spherical state appears. A principal indicaior of this is shown in Figure 2. In Fig. 2 the current collected on the anode, $I_{A}$, is shown as a function of $V$ with fixed $B$. The sharp peak is interpreted as follows. As $V$ is increased, the limiting equipotential of the trap changes from prolate, to spherical,to oblate. Electrons are energetically unable to reach the anode below some threshold voltage near $V_{o}$, consistent with negligible $I_{A}$ observed in this range. However, for $V>V_{o}$, no such energy consideration can explain the rapid drop of $I_{A}$ above the spherical point. Rather, the sharp resonance near (essentially at) $V=V_{o}$ must be associated with the formation of a special spherically symmetric state.

At the spherical point, electrons passing near the trap center are reflected back very near the center by the applied well. A focus may be formed if central space-charge deflects a portion of the injected beam away from prompt loss back through the injection hole. Electrons so deflected will reflex a number of times back near the center, providing a spherical core with which other electrons interact. Such a source is that required to form a focussed spherical state, as discussed subsequently, based on a numerical model of collisional evolution of the electron distribution (subsequent Section). The formation of a focussed state produces the space charge required to deflect injected electrons and maintain that focus, leading to a bootstrapping.

Additional diagnostics confirm the interpretation of spherical focussing. Figure 3 shows the detection of upscattered and downscattered electrons for a similar $V$ scan near a distinct spherical point. The coincidence of the two diagnostics suggests that the trapping process is an elastic one, with electron-electron interactions responsible for the energy changes. The same resonance width is indicated by all three diagnostics of Fig. 2 and 3. This resonance width increases weakly with $I_{i}$ from around 1 to a few percent of $V_{o}$.

The size of the focus may be estimated from the width of the spherical resonance peak. If $V$ is near but not exactly $V_{o}$, the bounce motion of an electron passing near $\rho=0$ will not return the electron to the same minimum $\rho$. Instead, the minimum $\rho$ will range from bounce to bounce. A "wobble" will be generated by the deviation of the well from sphericity. 
Standard perturbation (from the exactly spherical case) theory shows that the average (over all azimuths) deflection of an electron is proportional to the fractional deviation of $V$ from $V_{o}$. If this deflection is less that the core radius $r_{c}$ determined by the mean minimum radius of the confined electrons, the spherical state will be undisturbed. If this deflection exceeds the core radius, however, the spherical state will be strongly modified or lost. The width of the spherical resonance is about $1 \%$, suggesting that the convergence (ratio of $a$ to $r_{c}$ ) is of the order of 100:1.

Figure 4 shows a hysteresis associated with trapped charge. Two sequences of $I_{A}$ are shown vs. $I_{i}$ at a fixed spherical point $(B=0.0722 T, V=941 V)$. The discrete points are the results of cyclic measurements in which $I_{A}$ is recorded and the trap is emptied before $I_{i}$ is changed and a new measurement of $I_{A}$ is made. At a threshold value $\left(I_{i} \approx 150 n A\right), I_{A}$ increases dramatically. The continuous line represents a sequence of data obtained at the same spherical point, but with continuous operation, during which $I_{i}$ is gradually decreased. That is, the trap is not emptied between each measurement of $I_{A}$. At a much lower threshold $\left(I_{i} \approx 15 n A\right) I_{A}$ returns to its low value. This hysteresis is consistent with the pump beam producing a seed of thermal core plasma near $\rho=0$ whose space charge eventually becomes large enough to deflect a significant portion of the beam and form a spherical focus. A focus is maintained so long as the electron resident time (inversely proportional to $I_{i}$ ) is sufficiently short compared to a collision time but vanishes when $I_{i}$ becomes too small. The subsequent Section gives the details of this model.

The scaling of threshold $I_{i}$ is found to be linear with $V$. [1] A linear variation is found both when the upper cathode potential is such that the injected beam passes directly through the trap, or when it is made more negative so that the beam is reflected back toward the injector. For the case of reflection, for example, the threshold $I_{i}$ is found to be well described by

$$
I_{i}=1.6 \times 10^{-10} \mathrm{~V}
$$

The thermal inventory varies linearly only with $I_{i}$. It is shown in the next Section that 
the deflected fraction of the incoming beam is proportional to the ratio of this inventory to the Brillouin inventory $\bar{N}$, defined as that which fills the trap sphere with a Brillouin density, $\bar{n}$. Thus, the observed linear scaling of threshold $I_{i}$ with $\bar{n}$ or with $V$ is consistent with this picture.

Spherical focussing has been observed over the range of PFX operation. Figure 5 shows a summary of the observation of the three diagnostics over the range of $B$ and $V$, compared to the theoretical relation (3). As can be seen, the agreement is excellent, with focussing indicated only for points within $1 \%$ of the curve given by (3).

Finally, a few additional observations are mentioned. First [1], there are significant effects of increasing $I_{i}$. These include a broadening of the resonance and wave activity observed as induced fluctuations of the cathode voltages or currents. These phenomena may be associated with large space charge effects on the formation and maintainence of the spherical focus. For example, it may be that at values of $I_{i}$ far above the threshold value for spherical formation, there is some shielding of the applied well toward a spherical one, with associated resonance broadening and sensitivity of the focus to collective effects.

An additional observation is that the focus shown by the data here is degraded if the intrinsic field error of the device is made too large. In one series of experiments, the axial separation of the cathodes from the anode was decreased by $250 \mu \mathrm{m}$, introducing an error in the electrostatic field of about $5 \%$. Under this condition, no appreciable focussing was observed in any of the diagnostics. This seems to indicate that the intrinsic field error is of order $1 \%$ or less in PFX. The focus is also degraded if the injected beam is too asymmetric. During one experimental series, the emitter developed a lobed emission pattern and the focus was not obtained.

\section{THEORETICAL ANALYSIS}

Now, the hysteresis shown in Fig. 4 is considered theroetically. Evidently, two distinct states coexist over the range of $I_{i}$ between the high-to-low threshold (subsequently referred 
to as the "lower threshold") and the low-to-high threshold (subsequently the "upper threshold"). This region will be subsequently referred to as the "two-state region". These states are a beam-like state (lower $I_{A}$ in Fig. 4) and a spherical state (upper $I_{A}$ in Fig. 4).

In the beam-like state, electron motion is confined to a small cylindrical region near the system axis. As evidenced by negligible $I_{A}$, electrons move axially back and forth in the axial electrostatic well of the trap and are not deflected significantly.

In the spherical state, injected electrons are deflected by a sufficient angle in a single pass that they are unable to promptly exit the trap or intersect the lower cathode. Because this state exists only when the well is spherically symmetric and only for sufficient $I_{i}$, it must be that this state is spherically symmetric and is associated with space charge near the trap center causing a single pass, large angle deflection of injected electrons.

The consistency of this picture and the transition mechanisms are now considered. First, consider deflections of an injected paraxial beam due to space charge. It this deflection (per transit) is much less than the angle subtended by the injected beam at the trap center, the beam-like state will persist, since electrons are likely to be lost before they are significantly deflected from axial motion. A transition from the beam-like state will occur when deflection is comparable to this angle, or, equivalently, when the radial deflection is comparable to the beam radius.

It may next be seen that such a large deflection cannot occur as a result of the self-charge of the beam itself, since the beam charge density is much too low. Suppose, however, that a fraction of the injected current is trapped (for example, because some electrons are placed on strange orbits which do not easily intersect the electrodes) and such electrons are lost from the trap very slowly, over a period of several seconds. Such a long confinement time is consistent with the following observation.

In the two-state region the system is placed in the spherical state. Then, $I_{i}$ is interupted for a period by biasing the emitter positive. After such interuption, the system will return to the beam-like state, only if the interuption time is sufficiently long. This time is observed to be greater than 5 seconds in PFX. Such a time is much longer than the slowing-down time 
for a beam electron, but is consistent with expected intrinsic confinement time for thermal (energy $\ll$ well depth) electrons.

Combining the above elements, the upper threshold may be understood as follows. A very small fraction of the injected beam current becomes trapped, and that fraction is independent of $V$ (with sphericity maintained by scaling $B$ appropriately. These trapped electrons cool and form a thermal electron cloud, which is lost slowly to the anode by nonideal effects. Because the rotation rate of such a cloud (and hence the retarding torque) is due to $\mathbf{E} \times \mathbf{B}$ and is thus proportional to $B$, the cross- $B$ loss rate is independent of $B$ (correspondingly $V$ ). The inventory of trapped electrons is thus proportional to the injection current, independent of $V$.

The thermal electron cloud forms a $\bar{n}$ sphere at the bottom of the well near the trap center. If the radius of such a cloud is $\bar{r}$, the deflection of a paraxial electron is given by

$$
\ddot{r}=\frac{e^{2} \bar{n} \frac{4 \pi}{3} \bar{r}^{3}}{4 \pi \epsilon_{o} m} \frac{r}{\left(z^{2}+r^{2}\right)^{3 / 2}},
$$

where $\epsilon_{o}$ is the permitivity of free space, and $(r, z)$ the instantaneous cylindrical coordinates.

Equation (5) is integrated with several simplifying assumptions. First, the deflection is considered small, so that $r$ on the right of (5) is replaced by $b$, the initial value of $r$, the impact parameter. Second, the (nearly axial) velocity of the electron is considered to be constant. This is justified since most of the deflection occurs close to the equatorial plane, where the vacuum well is flat. Then (5) may be integrated twice in $z$ from $z=-a$ to $z=a$ to obtain

$$
r(z=a)-r(z=-a)=\frac{2 a e^{2} \bar{n} \bar{r}^{3}}{3 \epsilon_{o} m v^{2} b}
$$

where the constant velocity is $v$. Now defining the fractional deflection as $f=[r(z=$ $a)-r(z=-a)] / b$ and using that the deflected electron's energy is equal to the well depth, $f$ may be expressed in terms of the ratio of thermal inventory to Brillouin inventory,

$$
f=2 \frac{N}{\bar{N}}\left(\frac{a}{b}\right)^{2},
$$


where the Brillouin inventory $\bar{N}=4 \pi \bar{n} a^{3} / 3$ and the inventory is, of course, $N=4 \pi \bar{n} \bar{r}^{3} / 3$. Condition (7) is valid only so long as $b>\bar{r}$. For PFX, $a / b=30$, and if $\bar{r}=b, f=6 \%$, so a significant deflection occurs for these conditions, with $N / \bar{N}=3 \times 10^{-4}$.

Based on the preceeding arguement, the upper transition should occur when $N / \bar{N} \sim$ $10^{-3}$. If the intrinsic confinement time (that for thermal electrons) of the trap is estimated to be $1 s$, the scaling observed (4) is consistent with $4.2 \times 10^{-6}$ of the input beam current being trapped as thermal electrons in the beam state. Thus, a very small intrinsic beam trapping efficiency is required to explain the observed transition scaling.

Once the upper threshold is reached, the fraction of beam available to build up a core density rapidly increases. The thermal core electrons deflect beam electrons by a sufficient angle that they reflex many times back near $\rho=0$ and increase the space charge there, leading to a bootstrapping in which the fraction of deflected beam electrons rapidly reaches nearly unity. The spherical state is then maintained so long as the throughput of electrons is sufficient to produce a nonthermal, beam distribution. Maintaining such a nonthermal distribution requires that the resident time of a typical electron be less than the electronelectron collision time.

For the example of Fig. 4, the electron-electron collision time, based on the well depth energy and $\bar{n}$ is $5.8 \mathrm{~ms}$, while the resident time associated with the lower transition is $1.7 \mathrm{~ms}$, assuming that $N / \bar{N}=0.04$ consistent with previously computed, high convergence (small $\left.r_{c} / a\right)$ solutions. [5] Since the effective collision time is decreased by convergence [9], it is reasonable to suppose that the lower transition is associated with the throughput of electrons being insufficient to maintain the nonthermal focussed state against collisions. While the scaling of this transition with $V$ has not been carefully observed, the present analysis suggests that the lower threshold should scale as $V^{1 / 2}$.

To further analyze the spherical state, a fully nonlinear, bounce-averaged, Fokker-Planck code (BAFP) has been developed and applied to PFX conditions. This calculation combines several models to describe the complications presented by strong spherical convergence. Velocity-space diffusion is computed using the Rosenbluth form of the Fokker-Planck oper- 
ator [11], with the potentials found by first using the Green's function to get the potentials on a numerical boundary in velocity space and then finding the interior solution by Poisson solves using an iterative method [12]. To accomplish the bounce averaging, the unknown distribution function is first represented as a function of the constants of the motion for a spherically symmetric system, that is, the total energy and the total angular momentum. Next, this function is mapped at each of several spherical radii to cylindrical velocity space variables $\left(v_{r}, v_{\perp}\right)$. Part of this mapping is the determination of the self consistent electrostatic potential $\Phi$, using the number density (as a function of $\Phi$ ). Then the diffusion problem is solved at each radius to determine the collisional part of the time derivative of the distribution function. Finally, this time derivative is averaged along each orbit corresponding to a mesh of energy and angular momentum pairs, sources and sinks are added and the distribution is advanced to the next time step.

Once sources and sinks are specified, the time asymptotic solution is found by advancing time using BAFP until a steady state is observed. The resulting distribution completely determines the state of the system. To model PFX, a fictitious background density is added to represent the vacuum well. A cool electron source is provided centered on an energy $1 \%$ greater than the top of the vacuum well (experimentally this energy excess is of the order of $10 \mathrm{~V}$ to $20 \mathrm{~V}$ compared to well depths of $350 \mathrm{~V}$ to $7 \mathrm{kV}$ ). Electron loss is modelled by a loss rate which increases from zero at the top of the vacuum well to a large value, with the rate of increase corresponding roughly to the area intersected on the electrodes by ever higher equipotentials. This normalized (to the mean electron-electron collision time previously defined) rate of increase of the electron loss, along with the location of the electron source completely determines the solution.

An example of a steady solution found by BAFP is shown in Fig. 6, where the density and potential vs. spherical radius are plotted. The electron density is normalized to $\bar{n}$, the radius to $a$, and the potential to the well depth. Not only does the calculation confirm the formation of a steady focussed spherical state, but shows a strong density peaking. According to the model, an axial density of nearly $36 \bar{n}$ is produced in a spot of $30 \mu \mathrm{m}$ radius 
by spherical focussing.

\section{DISCUSSION AND CONCLUSIONS}

The present results, combined with a theoretical analysis indicate that PFX has achieved the desired spherical focus over a range of well depths. The indicators of this are: a sharp resonance whose width is expected to be comparable to the ratio of the core radius to the trap radius, indicating a convergence of 100:1; the sudden transition to large angle deflections of injected electrons, indicating the appearance of significant central space charge; expected scaling with $B, V$, and $I_{i}$; and sensitivity of obtaining the focus (resonance) to field and injection errors.

A numerical model has been developed and applied to compute the electron distribution produced in PFX. In this model, the electron distribution is assumed to depend only on the spherical radius and velocity and to evolve according to classical, small-angle collisions according to the fully nonlinear Fokker-Planck model. The radial effective potential includes the applied spherical well and the space charge of the confined electrons. Using sources and losses consistent with PFX parameters, it is found that a focussed state is formed and maintained with a convergence of order 100 . The core of this focus is of radius $30 \mu \mathrm{m}$ and has a density of $35 \bar{n}$. The consistency of the observations with this computed state suggests that the electron behavior in PFX is classical and that the Brillouin density has indeed been significantly exceeded by formation of a spherical focus.

Near-term improvements to PFX are focussing on two related areas for fusion relevance. First, the electron emitter is being relocated to a distance of a few $\mathrm{cm}$ below the lower cathode, and transfer optics are being added to propagate the beam to the lower cathode injection hole. It is anticipated that this will allow the electrodes to remain cold and raise the hold off voltage to perhaps as much a $30 \mathrm{kV}$. Second, a gas system is being installed on the machine as a first attempt to introduce ions into the virtual cathode formed by the central electron focus. The neutrals will be ionized by electron impact of the injected electrons and 
those formed within a sphere centered around the cathode at $\rho=0$ will be electrostatically trapped and heated by the energetic electrons. A low neutron signal may be obtained in this manner for $V>25 \mathrm{kV}$, according to various estimates.

In the longer term, an upgrade to PFX is being planned. This system will change the applied $\mathbf{B}$ and $\mathbf{E}$ to allow the confinement of a nearly uniform sphere of nonneutral electron plasma. These electrons will then form a virtual cathode from their space charge, and ions introduced at the outside of this uniform density sphere will have the same dynamics as the present PFX electrons, since they will find themselves in a spherical, harmonic well. Such a SCIF system is useful to test many of the physics issues of focussed ion systems in a very small device and will allow both higher well depths (by limiting the electrostatic stress away from narrow gaps, as in present PFX) and more efficient use of the well depth to produce ion energy. Thus, higher fusion rates may be expected, and it may be possible to approach the ultimate limiting values of $Q$. This would provide a very useful source of $14 \mathrm{MeV}$ neutrons in an extremely compact system.

In the still longer term, two critical issues must be addressed to demonstrate the usefulness of this fusion concept for large power applications. First, the system size must be increased beyond one or two Debye lengths. Second, a means must be found to avoid the large powers predicted to be required to maintain the desired nonthermal spherical convergence. $[8,9]$

Increasing the system size to the quasineutral limit introduces a number of new physics issues. The electrostatic stability of counterstreaming beams in a large system is not completely understood. The larger size means that measures must be taken to avoid the defocussing effects on $\mathbf{B}$ on converging ion motion. A new magnetic configuration is necessary to place all the vacuum well potential drop near the edge of the machine.

If $Q$ is limited to unacceptably low values because of ion-ion collisions, it will be necessary to explore new regimes of operation. A promising approach to circumvent the predicted limitations is to make use of the space charge of the ion focus to produce a "double well" of the type proposed by Hirsch. [6] In some recent BAFP calculations it has been observed that 
it is possible to produce an off axis peaking of the beam ions if their angular momentum is peaked away from zero. In the presence of Maxwellian electrons there will be an associated electrostatic potential minimum on the system axis. Within this central well, thermonuclear ions may be well confined. If this core region is consider the fusion source, the beam energy becomes decoupled from the fusion cross section, and $Q$ may be raised by increasing this beam enregy and lowering the collisionality of the nonthermal ion component. Such speculations will be tested in future work. First by improved theoretical models and computations, and then by small experiments.

\section{ACKNOWLEDGMENTS}

This work was sponsored by the Office of Fusion Energy Science of the U.S. Department of Energy. One of the authors (DCB) acknowledges supporting contributions from L. Chacon of U. Illinois, and R. Nebel, J. Finn, F. Ribe of Los Alamos.

\section{DISCLAIMER}

This report was prepared as an account of work sponsored by an agency of the United States Government. Neither the United States Government nor any agency thereof, nor any of their employees, makes any warranty, express or implied, or assumes any legal liability or responsibility for the accuracy, completeness, or usefulness of any information, apparatus, product, or process disclosed, or represents that its use would not infringe privately owned rights. Reference herein to any specific commercial product, process, or service by trade name, trademark, manufacturer, or otherwise does not necessarily constitute or imply its endorsement, recom$/$ mendation, or favoring by the United States Government or any agency thereof. The views and opinions of authors expressed herein do not necessarily state or reflect those of the United States Government ôr any agency thereof. 


\section{REFERENCES}

[1] T. B. Mitchell, M. M. Schauer, and D. C. Barnes, "Observations of Spherical Focus in an Electron Penning Trap", Phys. Rev. Lett., In Press (1996).

[2] M. M. Schauer, T. B. Mitchell, D. C. Barnes, and M. H. Holzscheiter, "An Electron Penning Trap for the Generation of High Density Nonneutral Plasmas", in preparation (1996).

[3] Non-neutral Plasma Physics, edited by C.W. Roberson and C.F. Driscoll (American Institute of Physics, New York, 1988); Non-neutral Plasma Physics II, edited by J. Fajans and D. H. E. Dubin (American Institute of Physics, New York, 1995); R. C. Davidson, Physics of Nonneutral Plasmas (Addison-Wesley, Redwood City, CA, 1990).

[4] L. Brillouin, Phys. Rev 67, 260 (1945).

[5] D. C. Barnes, R. A. Nebel, L. Turner, and T. N. Tiouririne, Plasma Phys. Control. Fusion 35, 929 (1993).

[6] R. L Hirsch, J. App. Phys. 38, 4522 (1967).

[7] R. W. Bussard, Fusion Technology 19, 273 (1991).

[8] Todd H. Rider, Phys. Plasmas 2, 1853 (1995).

[9] W. M. Nevins, Phys. Plasmas 2, 3804 (1995).

[10] L.S. Brown and G. Gabrielse, Rev. Mod. Phys. 58, 233 (1986).

[11] Marshall N. Rosenbluth, William M. MacDonald, and David L. Judd, Phys. Rev. 107, 1 (1957).

[12] R. D. Milroy, D. C. Barnes, R. C. Bishop, and R. B. Webster, Phys. Fluids B1, 1225 (1989). 


\section{FIGURES}

FIG. 1. PFX hyperbolic trap. The three electrodes are mechanically connected by ceramic spacers whose cathode ends are tailored to maximize voltage standoff and suspended by three support rods. An emitter is placed very near the injection hole in the lower cathode.

FIG. 2. Observed anode current vs. anode voltage, $V$, near the spherical point. As injection current is increased from the lower (injection current $\left.I_{i}=0.05 n A\right)$ to the higher $\left(I_{i}=0.8 n A\right.$ ) curve, a sharp resonance appears.

FIG. 3. Observed upscattered (crosses) and trapped (downscattered) electrons (squares) vs. $V$ near a second spherical point. These data are a coincident indication of an inelastic trapping process occuring only near the spherical point.

FIG. 4. Hysteresis curve for spherical trapping at fixed spherical point, $B=0.0722 T$, $V=941 V$. As $I_{i}$ is increased (abscissa), $I_{A}$ (ordinate) suddenly rises from a beam-like state to a spherical state. Hysteresis observed as $I_{i}$ is decreased is consistent with collective space charge trapping of injected beam electrons. Solid circles are individual filling cycles, while continuous curve is quasistatic decrease of $I_{i}$.

FIG. 5. Summary of three data indicative of spherical state. Location of maximum $I_{A}$ (squares), upscattered electrons (diamonds), and downscattered electrons (crosses) is shown vs. anode voltage $V$ and magnetic field $B$. Solid curve is the calculated theoretical spherical condition of Eq. (1).

FIG. 6. (a) Normalized (to $\bar{n}$ ) density predicted by BAFP code for PFX parameters. Maximum density is nearly 36 times $\bar{n}$, with an effective core radius $r_{c}$ of $1 \%$ of $a$ (30 $\mu m$ for PFX). (b) Normalized (to well depth $W$ ) effective potential predicted by BAFP. Virtual cathode associated with core space charge is nearly $25 \%$ of $W$ for this case. 


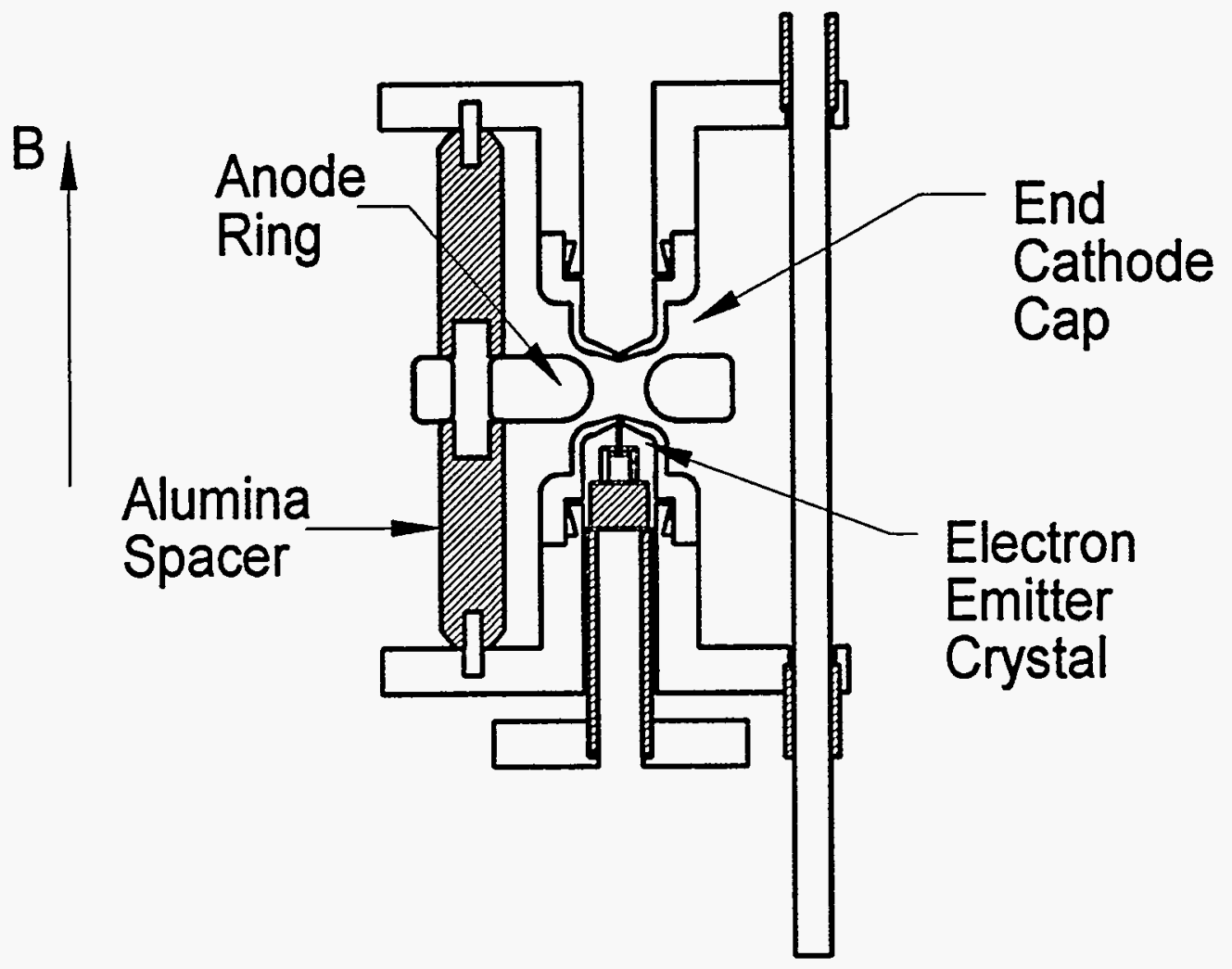

Fig. 1 


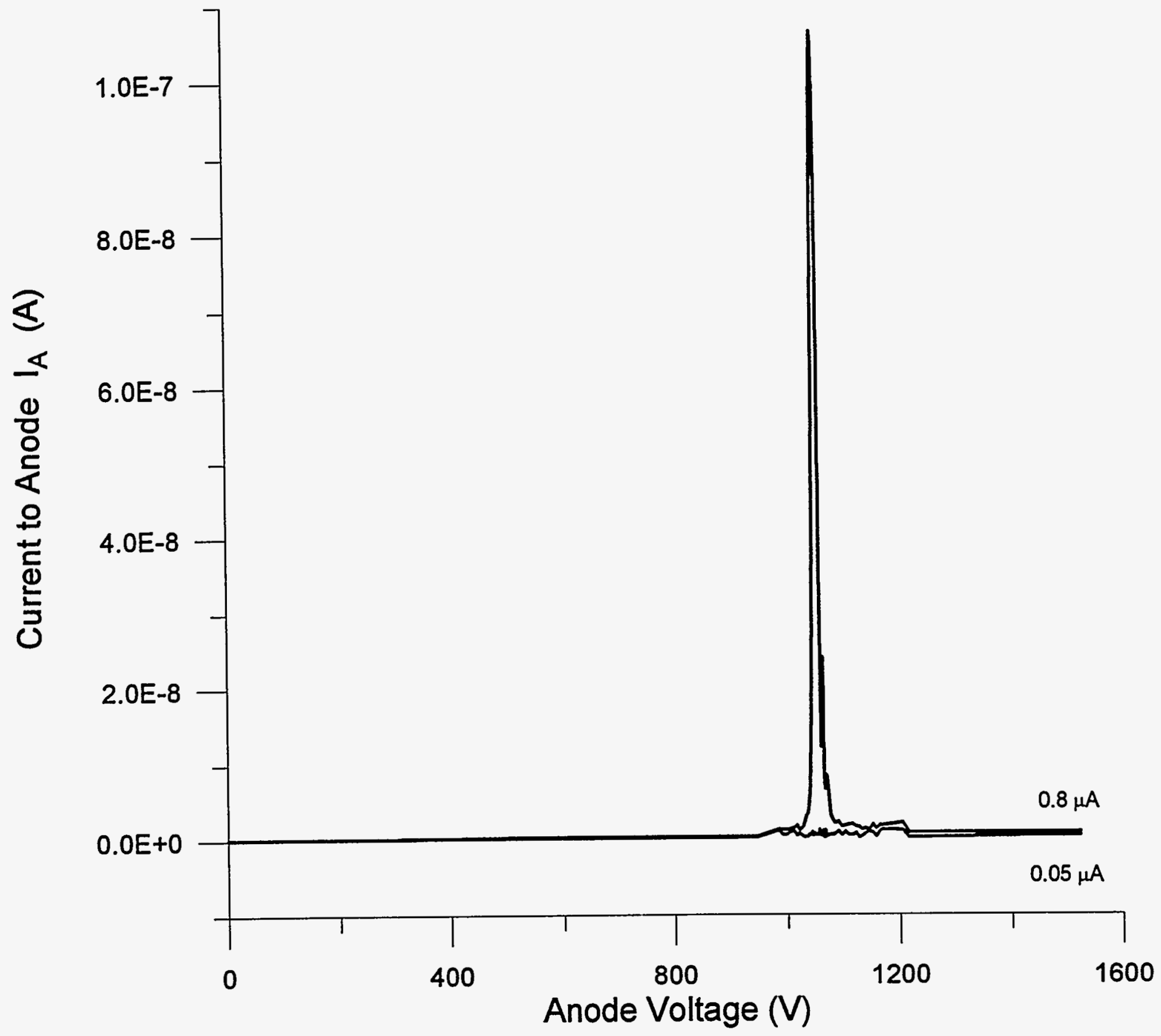

Fig. 2 


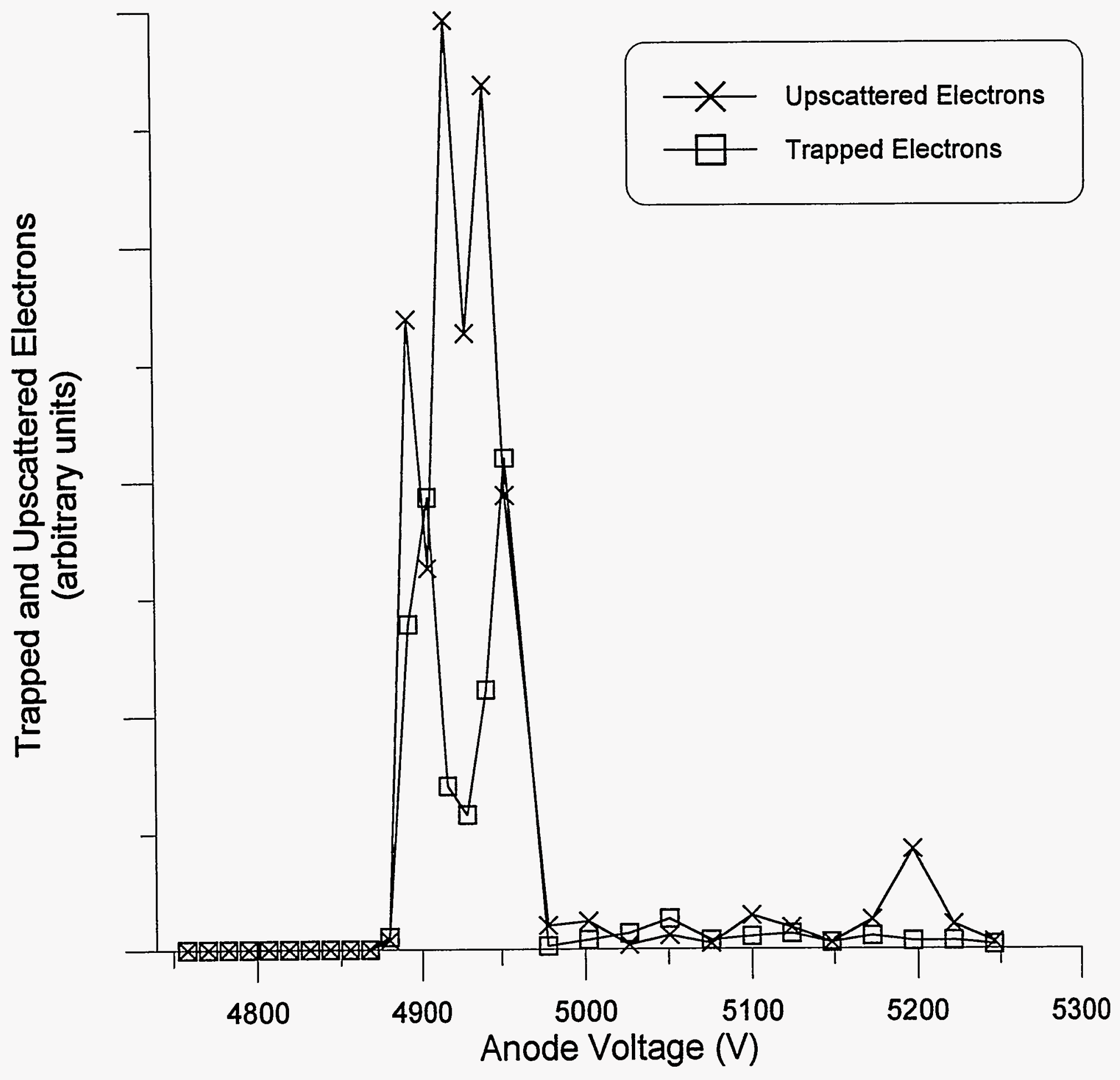

Fig. 3 


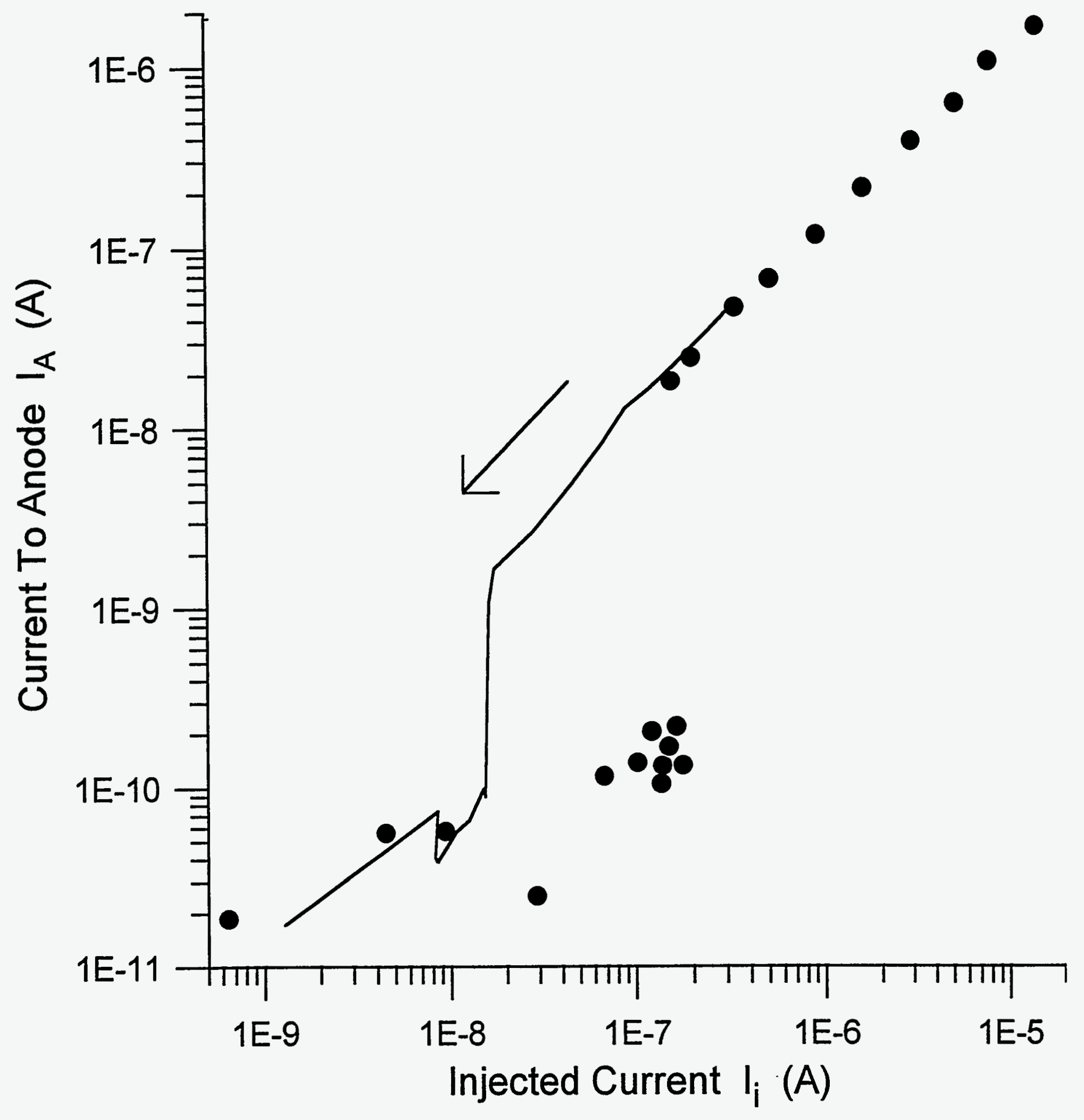

Fig. 4 


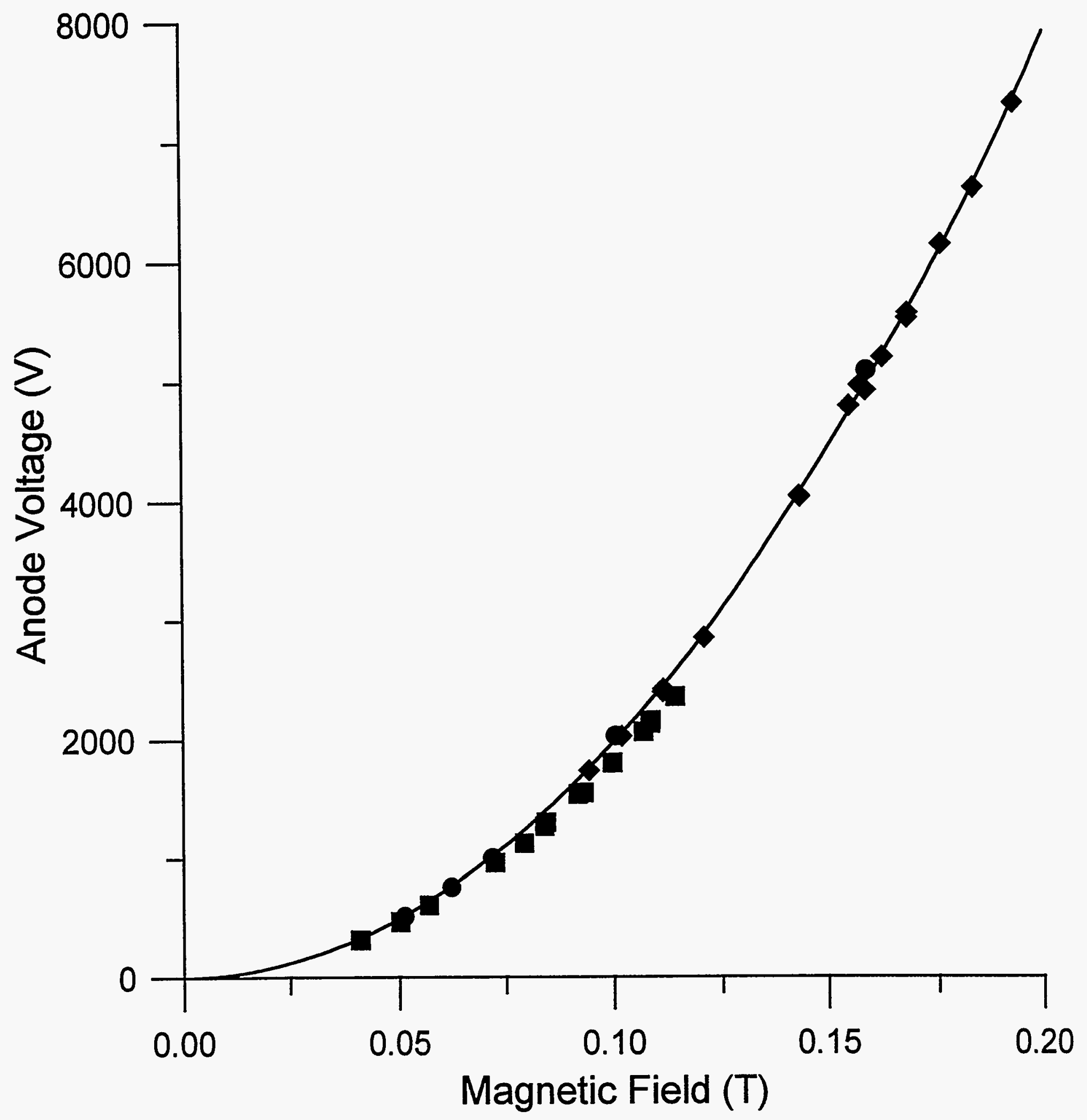

$F_{i z}, 5$ 


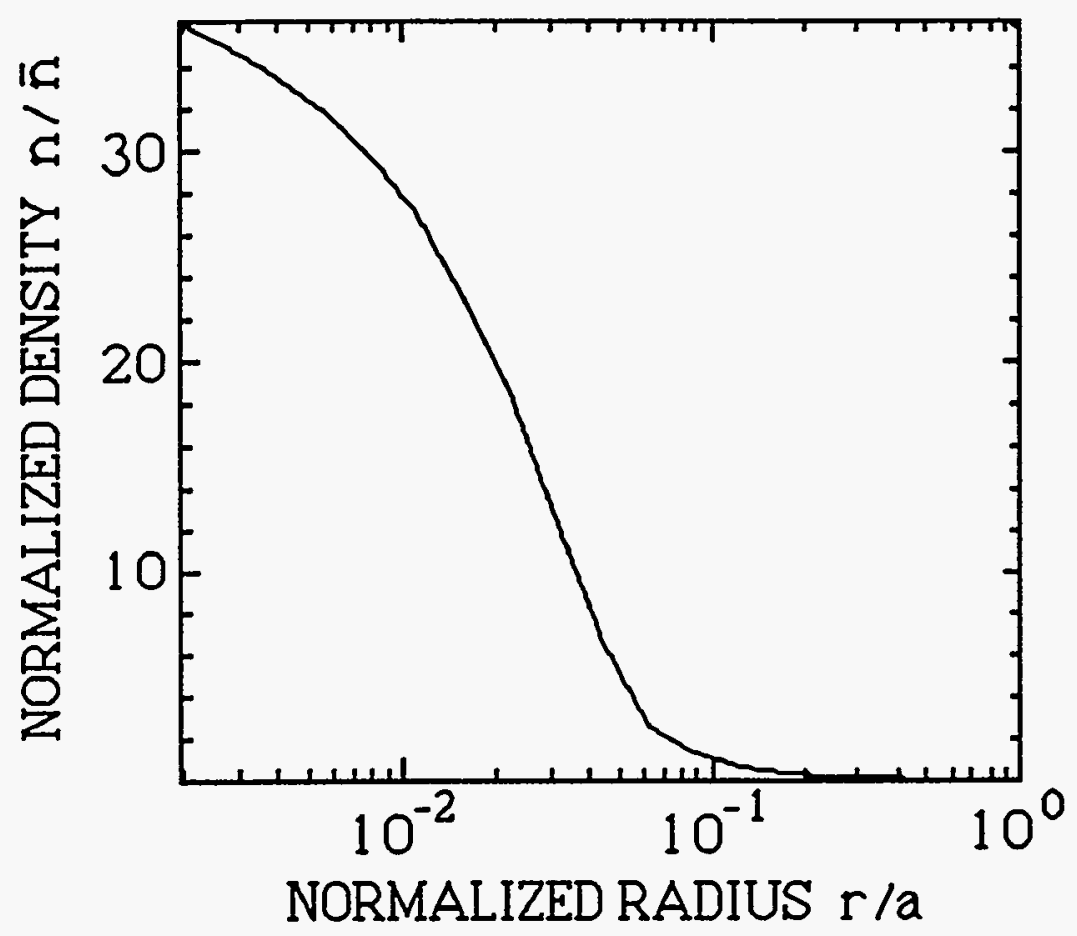

(a)

Fig. 6 (a) 
$\pi$
0
0
0

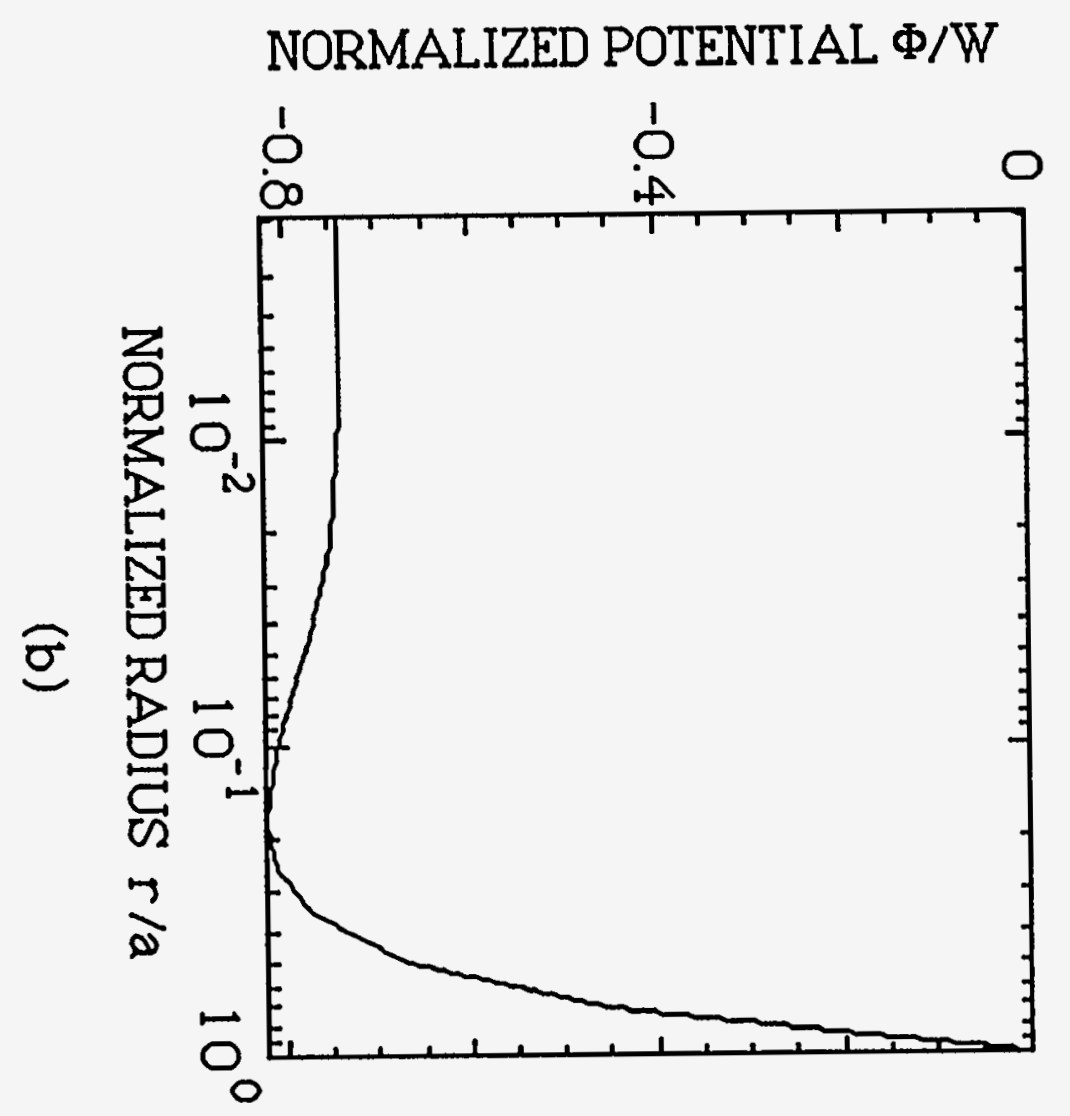

